\title{
Post-Saline Infusion Plasma Aldosterone Concentrations are Well Correlated with the Lateralized Ratio of Adrenal Venous Sampling in Patients of Primary
} Aldosteronism

\author{
Yuko Tsushima ${ }^{1,2}$, Ken Terui ${ }^{1,2^{*}}$, Koshi Makita ${ }^{1,2}$, Yuko Asari ${ }^{1,2}$, Noriko Ishigame ${ }^{1,2}$, Yuki Nakada ${ }^{1,2}$, Aya Sugiyama ${ }^{1,2}$, Shingo Murasawa ${ }^{1,2}$, Satoshi \\ Yamagata $^{1,2}$, Yutaka Watanuki ${ }^{1,2}$, Shinobu Takayasu ${ }^{1,2}$, Takeshi Nigawara ${ }^{1,2}$, Kazunori Kageyama ${ }^{1,2}$ and Makoto Daimon ${ }^{1,2}$ \\ ${ }^{1}$ Department of Endocrinology and Metabolism, Hirosaki University Graduate School of Medicine, Hirosaki, Japan \\ 2Department of Endocrinology, Diabetes and Metabolism, Hirosaki University School of Medicine and Hospital, Hirosaki, Japan \\ *Corresponding author: Ken Terui, Department of Endocrinology and Metabolism, Hirosaki University Graduate School of Medicine, 5 Zaifu-cho, Hirosaki, Japan, Tel: \\ +81-172-39-5062, Fax: +81-172-39-5063, E-mail: terui@hirosaki-u.ac.jp
}

Received date: June 25, 2017; Accepted date: June 27, 2017; Published date: June 30, 2017

Copyright: @ 2017 Tsushima Y, et al. This is an open-access article distributed under the terms of the Creative Commons Attribution License, which permits unrestricted use, distribution, and reproduction in any medium, provided the original author and source are credited.

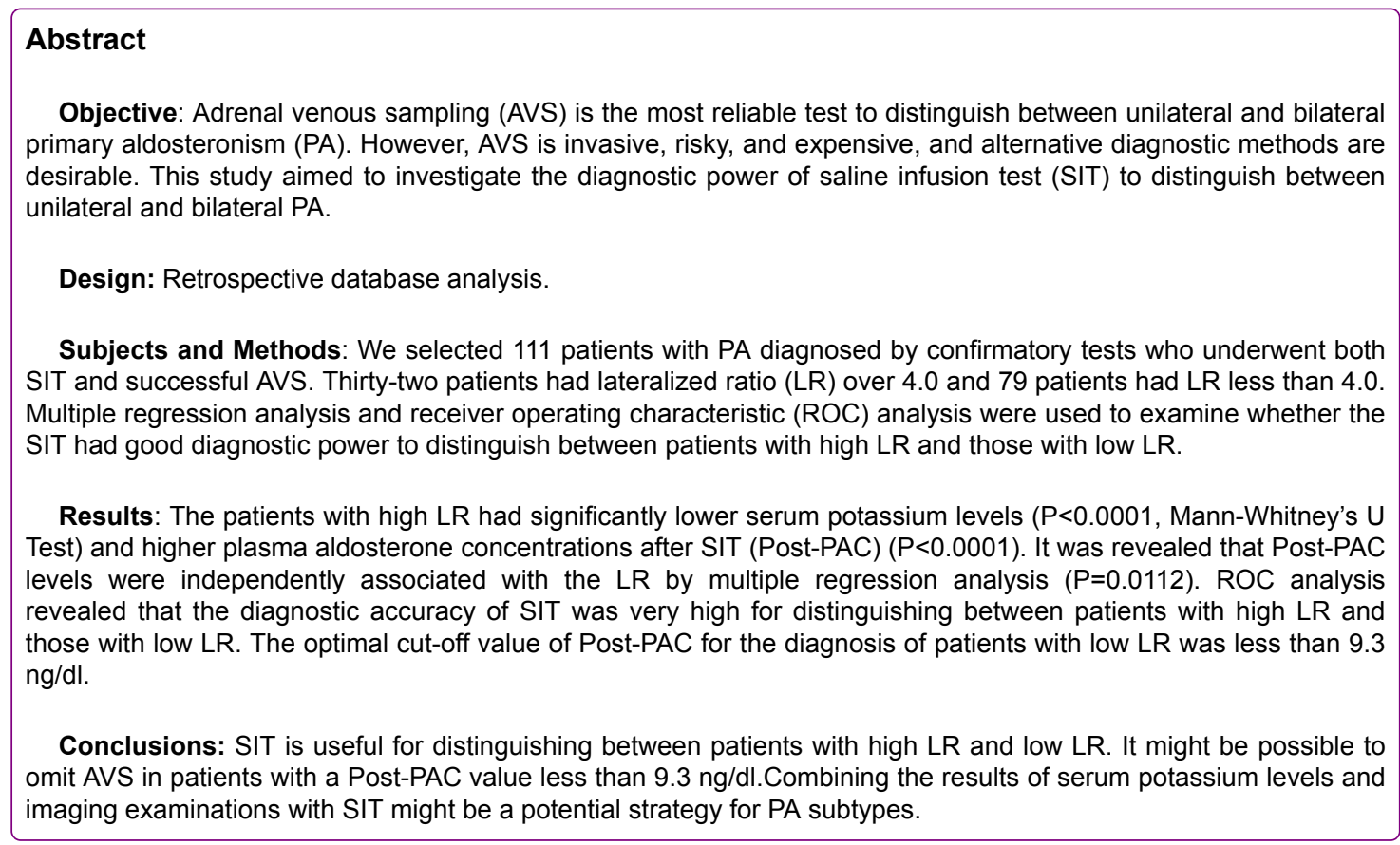

Keywords: Primary aldosteronism; Saline infusion test; Aldosteronerenin ratio; Adrenal venous sampling; Lateralized ratio

\section{Introduction}

Primary aldosteronism (PA) is currently considered to be the most frequent cause of secondary hypertension $[1,2]$. To screen for PA, the plasma aldosterone to renin ratio (ARR) is assessed in hypertensive patients [3]. If the ARR is higher than normal limits, confirmatory tests are requested, such as captopril challenge test (CCT), saline infusion test (SIT), fludrocortisone suppression test, and oral sodium loading test.

The Endocrine Society advocates the use of the four confirmatory tests in their clinical practice guidelines for PA. A conclusive diagnosis of PA is reached based on at least one positive test result $[3,4]$. The Japan Endocrine Society proposes the use of three confirmatory tests, that is, CCT, SIT, and furosemide plus upright test (FUT) [5]. Based on these guidelines, two positive test results are needed for diagnosis.
There is no consensus whether any confirmatory test is a gold standard for diagnosis.

After PA is diagnosed, the subtype should be identified in order to select the appropriate treatment for PA: surgical or medical treatment. In aldosterone-producing adenoma (APA) patients, hypersecretion of aldosterone is usually one-sided, that is, from one of the adrenal glands [6]. In patients with idiopathic hyperaldosteronism (IHA), hypersecretion is usually from both adrenal glands. Currently, adrenal venous sampling (AVS) is the most reliable test to distinguish between unilateral and bilateral PA [7,8]. To identify patients with PA, AVS is a reliable but invasive, risky, and costly procedure. 7 Therefore, alternative diagnostic methods are needed. A new guideline of the Endocrine Society recommends medical treatment without AVS if a patient is unable or unwilling to undergo surgery [4].

Küpers et al. proposed a clinical prediction score to diagnose unilateral PA. Their results suggest that AVS can be omitted in patients with a typical APA of at least $8 \mathrm{~mm}$ on computed tomography (CT), 
Citation: Tsushima Y, Terui K, Makita K, Asari Y, Ishigame N, et al. (2017) Post-Saline Infusion Plasma Aldosterone Concentrations are Well Correlated with the Lateralized Ratio of Adrenal Venous Sampling in Patients of Primary Aldosteronism. J Steroids Horm Sci 8: 186. doi:10.4172/2157-7536.1000186

Page 2 of 6

hypokalemia, and/or a high estimated glomerular filtration rate (eGFR) [9].

Arteaga et al. reported that SIT was useful for the differentiation of patients with APA from those with IHA [10]. Nanba et al. proposed that the shortened SIT be used to predict unilateral PA in Japanese patients [11]. Nevertheless, both these studies involved small patient populations.

The present study was performed to test whether the standard SIT has good diagnostic power for distinguishing between patients with unilateral and bilateral PA. To examine this hypothesis, we determined the best cut-off value to evaluate the diagnostic efficiency of SIT.

\section{Patients and Methods}

\section{Patients}

We retrospectively analyzed hypertensive patients with elevated ARR [ $>20 ;$ PAC in ng/dl and plasma renin activity (PRA) in $\mathrm{ng} / \mathrm{ml} / \mathrm{h}$ ] $(n=323)$ who were admitted to the Department of Endocrinology, Diabetes and Metabolism of Hirosaki University School of Medicine and Hospital from 2008 to 2016.

Before determination of ARR, mineralocorticoid receptor blockers, diuretics, beta-adrenergic blockers, angiotensin converting enzyme inhibitors and angiotensin receptor blockers were withdrawn for at least 2 weeks. These drugs should be changed to other drugs such as calcium channel blockers and alpha-adrenergic blockers. According to the Japanese guidelines, all of the patients had at least two of the three confirmatory tests for diagnosis of PA (CCT, SIT, or FUT). In this study, we re-evaluated the diagnosis of PA by CCA or FUT only. Overall, 295 patients had at least one positive test result of CCA or FUT, while 28 had negative results for all two tests. Of the 295 patients with positive test results, 278 underwent AVS, while the remaining 17 patients did not undergo AVS because they opted against surgery. AVS was successfully performed in 209 patients. This study involved 111 patients who underwent both SIT and successful AVS (Figure 1).

Hypokalemic patients were given adequate oral potassium supplements before SIT to maintain serum potassium levels higher than $3.0 \mathrm{mmol} / \mathrm{L}$. SIT was not performed in patients with severe uncontrolled hypertension, cardiac arrhythmia, and cardiac or renal insufficiency. The study was approved and registered as an institutional case-notes review at the Institutional Review Board of Hirosaki University School of Medicine and Hospital (Registration No. 2015-252).

\section{Saline infusion test}

Patients were administered an intravenous drip infusion of $2 \mathrm{~L}$ of $0.9 \mathrm{NaCl}$ over $4 \mathrm{~h}$. After infusion, the PAC (Post-PAC) was measured $[5,12]$.

\section{Adrenal venous sampling}

AVS was performed by expert radiologists to diagnose whether a unilateral or bilateral aldosterone-producing lesion was present. AVS was performed with cosyntropin infusion, as previously reported [13]. The lateralized ratio (LR) was calculated as (adrenal venous blood aldosterone/cortisol ratio on the high-value side)/(adrenal venous blood aldosterone/cortisol ratio on the low-value side). The contralateral ratio (CLR) was also calculated as (adrenal venous blood aldosterone/cortisol ratio on the low-value side)/(venous blood aldosterone/cortisol ratio in the inferior vena cava).

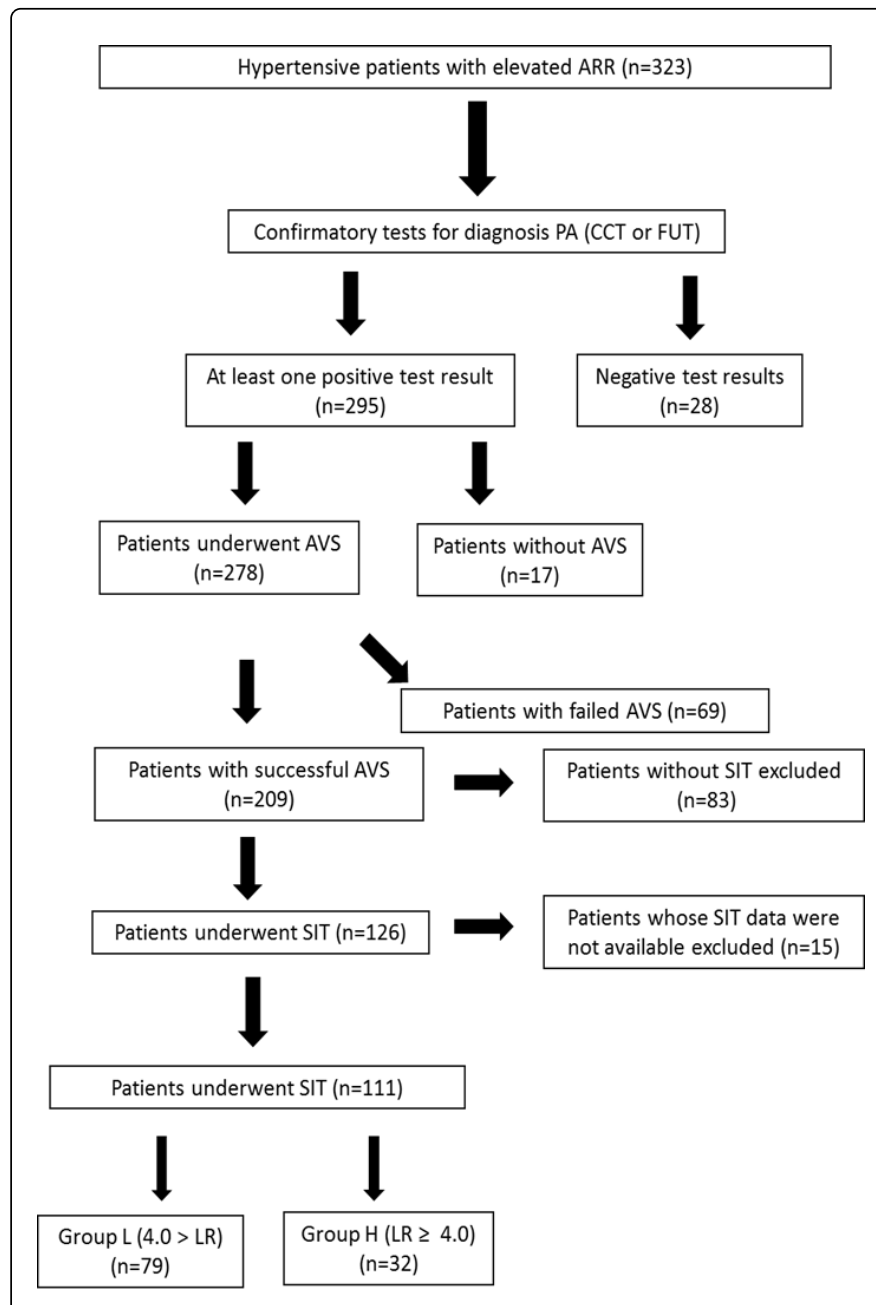

Figure 1: Flowchart of the study population. ARR: Plasma Aldosterone to Renin Ratio; PA: Primary Aldosteronism; AVS: Adrenal Venous Sampling; CCT: Captopril Challenge Test; FUT: Furosemide Plus Upright Test; SIT: Saline Infusion Test.

\section{Statistical analysis}

All the data are presented as medians with interquartile ranges. We used Mann-Whitney's U Test to compare medians between unilateral and bilateral groups. $\mathrm{P}<0.05$ was considered to indicate statistical significance.

Spearman's rank-order correlation analysis was used to examine the relationship between the clinical parameters and LR.

A multiple regression analysis was performed for correlation between LR as a dependent variable and clinical parameters including Post-PAC as independent variables. Because the data had a nonnormal distribution, logarithmic conversion was carried out. Factors with $P$ values less than 0.05 on univariate analyses were considered for inclusion in the model, and forward selection and backward elimination methods were used to choose the explanatory variables. 
Citation: Tsushima Y, Terui K, Makita K, Asari Y, Ishigame N, et al. (2017) Post-Saline Infusion Plasma Aldosterone Concentrations are Well Correlated with the Lateralized Ratio of Adrenal Venous Sampling in Patients of Primary Aldosteronism. J Steroids Horm Sci 8: 186. doi:10.4172/2157-7536.1000186

Page 3 of 6

The diagnostic accuracy of the SIT for differential diagnoses between bilateral and unilateral PA groups was assessed using the receiver operating characteristic (ROC) curve and the area under the ROC curve (AUC ROC .

\section{Results}

\section{Characteristics of the study population}

We classified all the patients with PA in one group had LR over 4.0 (group H) or the other group (group L). The clinical characteristics of the two groups are summarized in Table 1. The number of patients of group L was higher than that of patients of group H. Age, height, body weight, and body mass index showed no significant differences between the two groups. Serum potassium levels were significantly lower in patients of group $\mathrm{H}$ than in those of group L. Basal PAC levels and ARR in patients of group $\mathrm{H}$ were higher than those in patients of group L. Post-PAC values were significantly higher in patients of group $\mathrm{H}$ than in those of group $\mathrm{L}$.

\begin{tabular}{|c|c|c|c|c|}
\hline Variables & Total & Group L (4.0> LR) & Group H $(L R \geq 4.0)$ & $P$ value \\
\hline$N(M / F)$ & $111(44 / 67)$ & $79(27 / 52)$ & $32(17 / 15)$ & 0.0645 \\
\hline Age (year) [median (IQR)] & $53.0[46.0-61.0]$ & $53.0[48.0-61.0]$ & $58.0[46.0-65.0]$ & 0.1527 \\
\hline Height $(\mathrm{cm})$ [median (IQR)] & 159.6 [153.0-168.0] & $158.8[152.5-166.7]$ & 164.8 [158.1-168.9] & 0.0881 \\
\hline Body weight (kg) [median (IQR)] & $65.9[55.5-75.9]$ & $63.5[55.1-75.7]$ & $67.6[55.9-78.5]$ & 0.4983 \\
\hline BMI $\left(\mathrm{kg} / \mathrm{m}^{2}\right)[$ median (IQR)] & $25.6[22.1-28.2]$ & $25.6[22.3-28.6]$ & $24.6[22.0-28.1]$ & 0.9046 \\
\hline Serum potassium (mmol/l) [median (IQR)] & $3.7[3.3-3.9]$ & $3.8[3.6-4.0]$ & $3.1[2.5-3.4]$ & $<0.0001$ \\
\hline eGFR (ml/min/1.73 m²) [median (IQR)] & $84.1[71.0-96.2]$ & 84.9 [73-95.3] & $85.2[68.5-101.7]$ & 0.792 \\
\hline Basal PAC (ng/dl) [median (IQR)] & $145.5[11.4-23.0]$ & $13.4[10.4-17.8]$ & 26.1 [18.9-39.8] & $<0.0001$ \\
\hline Basal PRA (ng/ml/hr) [median (IQR)] & $0.3[0.2-0.4]$ & $0.3[0.2-0.4]$ & $0.2[0.1-0.4]$ & 0.1215 \\
\hline ARR [median (IQR)] & 59 [36-117] & $49[33-86]$ & $133[71-230]$ & $<0.0001$ \\
\hline Post-PAC (ng/dl) [median (IQR)] & $8.9[6.5-14.5]$ & 7.9 [5.9-9.2] & 26.2 [18.9-39.8] & $<0.0001$ \\
\hline Post-PRA (ng/ml/hr) [median (IQR)] & $0.2[0.1-0.3]$ & $0.2[0.1-0.3]$ & $0.2[0.1-0.3]$ & 0.3919 \\
\hline Contra-lateralized ratio [median (IQR)] & $1.4[0.7-2.1]$ & $1.8[1.3-2.2]$ & $0.4[0.3-0.6]$ & $<0.0001$ \\
\hline Lateralized ratio [median (IQR)] & $1.8[1.3-5.2]$ & $1.5[1.2-2.0]$ & \multicolumn{2}{|l|}{$10.4[7.0-17.4]$} \\
\hline
\end{tabular}

Table 1: Characteristics of the study population.

\section{Univariate and multiple regression analyses of continuous variables for LR}

Univariate correlation analysis between each continuous variable and LR was performed (Table 2). Serum potassium, basal PAC, basal ARR, and Post-PAC (Figure 2) were positively correlated with LR, whereas eGFR was not.

\begin{tabular}{|l|l|l|}
\hline Variables & Correlation coefficient & P value \\
\hline Age & -0.0438 & 0.6478 \\
\hline Height & 0.1809 & 0.0575 \\
\hline Body weight & 0.029 & 0.7628 \\
\hline BMI & -0.0747 & 0.436 \\
\hline eGFR & -0.0875 & 0.3611 \\
\hline Serum potassium & -0.5032 & $<0.001$ \\
\hline Basal PAC & 0.5714 & $<0.001$ \\
\hline Basal PRA & -0.1694 & 0.0755 \\
\hline
\end{tabular}

\begin{tabular}{|l|l|l|}
\hline Basal ARR & 0.4936 & $<0.001$ \\
\hline Post-PAC & 0.6414 & $<0.001$ \\
\hline Post-PRA & 0.6414 & 0.8677 \\
\hline Contra-lateralized ratio & -0.757 & $<0.001$ \\
\hline
\end{tabular}

Table 2: Univariate analysis of continuous variables for lateralized ratio.

To further examine whether these parameters were associated with LR, stepwise multiple linear regression analysis was performed. PostPAC and serum potassium levels were independently associated with LR among the factors analyzed (Table 3).

\section{Diagnostic accuracy of SIT}

The Post-PAC values were significantly lower in group $L$ than in group $\mathrm{H}$ (Figure 3). 
Citation: Tsushima Y, Terui K, Makita K, Asari Y, Ishigame N, et al. (2017) Post-Saline Infusion Plasma Aldosterone Concentrations are Well Correlated with the Lateralized Ratio of Adrenal Venous Sampling in Patients of Primary Aldosteronism. J Steroids Horm Sci 8: 186. doi:10.4172/2157-7536.1000186

Page 4 of 6

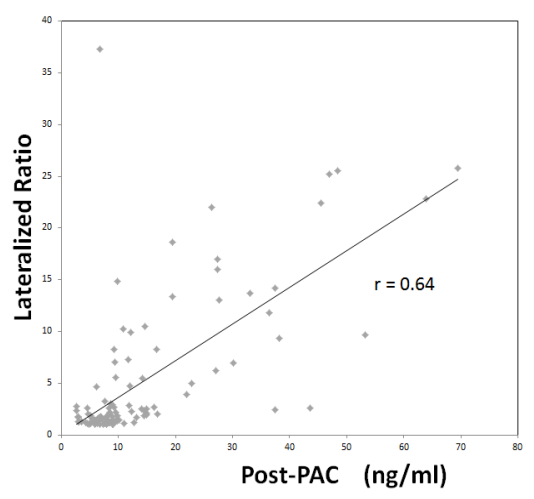

Figure 2: Relationship between plasma aldosterone concentration after saline infusion tests (Post-PAC) and the lateralized ratio (LR) of adrenal venous sampling.

\begin{tabular}{|l|l|l|l|}
\hline Variables & PRC & SPRC & P value \\
\hline Serum potassium & -1.629 & -0.2988 & $<0.001$ \\
\hline Basal PAC & 0.2173 & 0.1168 & 0.414 \\
\hline Basal ARR & 0.1158 & 0.0966 & 0.2896 \\
\hline Post-PAC & 0.4947 & 0.36 & 0.0112 \\
\hline
\end{tabular}

PRC: partial regression coefficient; SPRC: standardized partial regression coefficient

Table 3: Multiple linear regression analysis for lateralized ratio.

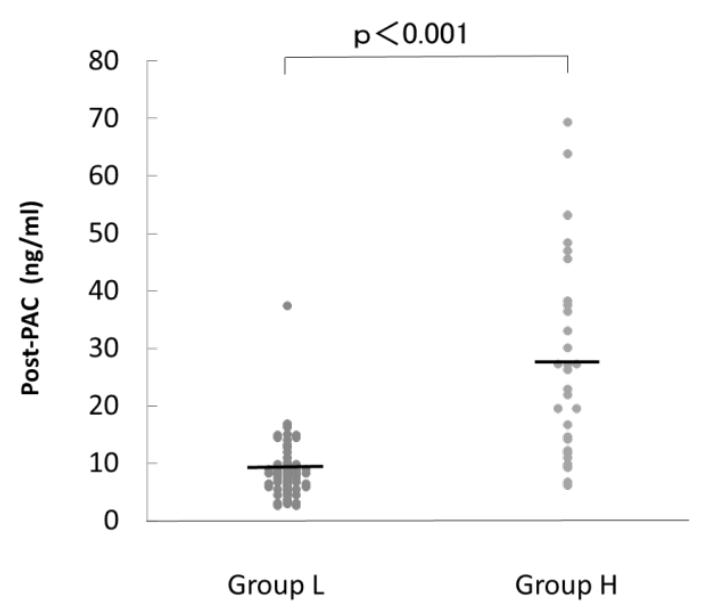

Figure 3: Scattergram of plasma aldosterone concentrations after saline infusion tests (Post-PAC) in group $\mathrm{L}$ and group $\mathrm{H}$.

We analyzed the diagnostic accuracy of the SIT for differential diagnosis between patients with group $\mathrm{L}$ and group $\mathrm{H}$. $\mathrm{AUC}_{\mathrm{ROC}}$ was approximately 0.9 , and the optimal cut-off value of Post-PAC was $<9.3$ $\mathrm{ng} / \mathrm{dl}$. These results showed a sensitivity of $76 \%$ and specificity of $94 \%$, respectively (Figure 4). The Youden index identified the same optimal cut-off value.

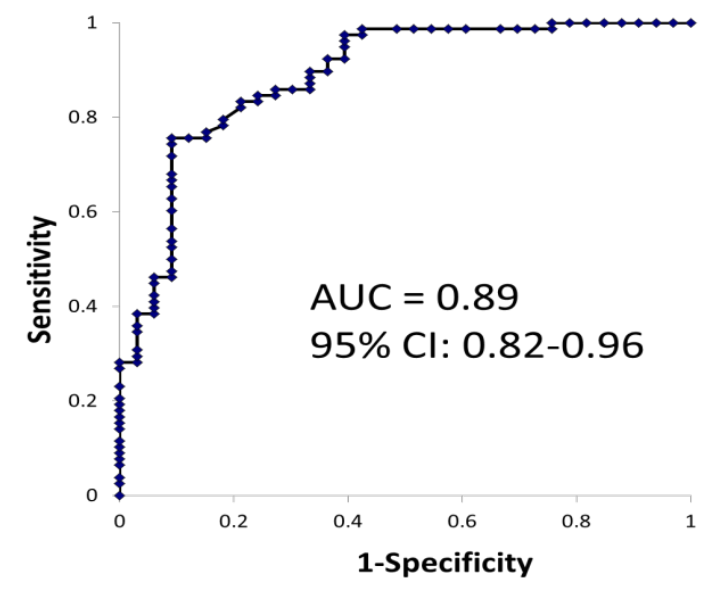

Figure 4: Receiver operating characteristic curves for plasma aldosterone concentrations after saline infusion tests (Post-PAC) for differential diagnosis between group $\mathrm{L}$ and group $\mathrm{H}$.

\section{Discussion}

After a diagnosis of PA, the subtype should be identified. While AVS is the most reliable test to distinguish between unilateral and bilateral $\mathrm{PA}$ and is used to make management decisions [7,12], AVS is an invasive, risky, and costly procedure [7,9]. The need for an alternative diagnostic method has posed a challenging problem in this field.

Küpers et al. proposed a clinical prediction score to diagnose unilateral PA [9]. Their results suggest that AVS could be omitted in patients with a typical APA measuring at least $8 \mathrm{~mm}$, with serum potassium levels $<3.5 \mathrm{mmol} / \mathrm{L}$ and/or eGFR of at least $100 \mathrm{ml} / \mathrm{min} / 1.73$ $\mathrm{m}^{2}$. In our findings, serum potassium levels were also positively correlated with LR by univariate correlation analysis. In contrast, eGFR was not correlated with LR. Riester et al. reported that the prediction score proposed by Küpers et al. has high accuracy only in young patients but cannot substitute for AVS in elderly patients [14]. Furthermore, in the present study, stepwise multiple linear regression analysis revealed that Post-PAC of SIT and serum potassium levels were independently associated with LR among the analyzed parameters.

Some papers have reported that confirmatory tests for PA are useful for distinguishing between unilateral and bilateral PA. Jiang et al. reported that the ACTH stimulation test with $1 \mathrm{mg}$ dexamethasone was useful for differentiating patients with unilateral PA from patients with bilateral PA [15]. We reported that ACTH stimulation test suggest that there is a high likelihood of laterlizing on AVS [16]. Arteaga et al. reported that SIT and CT scan were useful in the differentiation of patients with APA from those with IHA [10]. They evaluated 20 patients with PA, 14 patients with APA who were surgically treated, and 6 patients with IHA. In the SIT method used in their study, the patients were administered $1.25 \mathrm{~L}$ of $0.9 \%$ saline solution over $2 \mathrm{~h}$. They reported that after infusion, a PAC/cortisol ratio of $<2.2$ was diagnostic of IHA. On the other hand, Magill et al. reported that CT 
Citation: Tsushima Y, Terui K, Makita K, Asari Y, Ishigame N, et al. (2017) Post-Saline Infusion Plasma Aldosterone Concentrations are Well Correlated with the Lateralized Ratio of Adrenal Venous Sampling in Patients of Primary Aldosteronism. J Steroids Horm Sci 8: 186. doi:10.4172/2157-7536.1000186

Page 5 of 6

imaging was unreliable for the differentiation of PA and that AVS was essential to identify the PA subtype [17].

Nanba et al. [11] reported the shortened SIT method, wherein patients were administered $2 \mathrm{~L}$ of $0.9 \%$ saline solution over $4 \mathrm{~h}$ as usual; they proposed that PAC at $2 \mathrm{~h}$ would be more useful for predicting unilateral PA. They evaluated 39 patients with PA, including 18 patients with unilateral PA and 21 patients with bilateral PA. In their paper, the optimal cut-off value of PAC at $2 \mathrm{~h}$ was greater than $119 \mathrm{pg} / \mathrm{ml}$ and the $\mathrm{AUC}_{\mathrm{ROC}}$ was about 0.89 , with a sensitivity of $89 \%$ and specificity of $81 \%$. Recently, the importance of SIT as a confirmatory test for PA has become controversial. Weigel et al. reported that SIT was the most widely used confirmatory test for PA in Germany, due to practicability, costs, and compliance [18]. On the other hand, Nanba et al. investigated the diagnostic significance of confirmatory tests in Japanese hypertensive patients [12] and reported that the positive rate on SIT was low, being $<65 \%$. Li et al. reported that PAC after SIT remained higher than $5 \mathrm{ng} / \mathrm{dl}$ in a great majority of Chinese patients with essential hypertension as well as in healthy subjects [19]. Thus, it seems that SIT lacks adequate efficacy as a confirmatory test for PA but has diagnostic power for differentiation of PA subtypes in Asian patients.

In the present study, we found that standard SIT was useful for differentiating between patients with low LR and high LR in a larger population of patients than those assessed in the two above-mentioned papers concerning SIT $[10,11]$. The optimal cut-off value of Post-PAC was $<9.3 \mathrm{ng} / \mathrm{dl}$, with a very high accuracy for the diagnosis of low LR patients. Our findings suggest that AVS can be omitted in patients with Post-PAC $<9.3 \mathrm{ng} / \mathrm{dl}$ in standard SIT. For these patients, medical treatment may be selected directly without further invasive tests. Patients with Post-PAC of $9.3 \mathrm{ng} / \mathrm{dl}$ and above in standard SIT are likely to have unilateral PA; therefore, AVS is needed for these patients. Omitting AVS for approximately half of the patients with PA based on SIT results may enable a reduction in the risks and costs of the timeconsuming AVS procedure.

Some limitations of our study should be mentioned. This study was a retrospective analysis, and recall bias cannot be excluded. In order to diagnose PA in our patients, we used the diagnostic criteria of Japanese guidelines for Japanese patients. Additional studies are needed for clarifying the usefulness of the standard SIT in other populations. Recently, a French group reported that SIT had a good predictive value for unilateral PA; however, $30 \%$ of patients with Post-PAC $<5 \mathrm{ng} / \mathrm{dl}$ in SIT had unilateral secretion of aldosterone by AVS results [20]. These results disagree with our findings, that is, the PAC after SIT was over $9.3 \mathrm{ng} / \mathrm{dl}$ in almost all patients (94\%) with high LR in our study. The reason underlying this difference may be the use of different AVS methods in the two studies and the presence or absence of ACTH stimulation [21]. While we used the cut-off value of the LR that was at least 4.0, more permissive cut-off value of LR was used in many previous studies. Because Umakoshi et al. reported that such permissive cut-off values could be seen even in non-PA patiets [22], we used a cut-off value of LR with a high specificity for unilateral PA patients.

Considering our findings alone, approximately $6 \%$ of patients with high LR would not undergo AVS and subsequent surgical treatment. Recently, Dekkers et al. reported that treatment of PA based on CT scan or AVS findings did not show significant differences in the intensity of medical treatment or clinical benefits [23]. Combining the results of serum potassium levels and imaging examinations such as
CT with SIT might be a potential strategy for differentiating between patients with unilateral and bilateral PA.

In conclusion, standard SIT was useful for distinguishing between patients with low LR and high LR. Post-PAC of SIT and serum potassium levels were closely related to LR than other parameters, including eGFR. For the diagnosis of patients with low LR, the optimal cut-off value of Post-PAC was less than $9.3 \mathrm{ng} / \mathrm{dl}$. Accordingly, combining the results of serum potassium levels and imaging examinations with SIT might be a potential strategy for PA subtypes.

\section{References}

1. Williams JS, Williams GH, Raji A, Jeunemaitre X, Brown NJ, et al. (2006) Prevalence of primary hyperaldosteronism in mild to moderate hypertension without hypokalaemia. J Hum Hypertens 20: 129-136.

2. Rossi GP, Bernini G, Caliumi C, Desideri G, Fabris B, et al. (2006) A prospective study of the prevalence of primary aldosteronism in 1,125 hypertensive patients. J Am Coll Cardiol 48: 2293-2300.

3. Funder JW, Carey RM, Fardella C, Gomez-Sanchez CE, Mantero F, et al. (2008) Case detection, diagnosis, and treatment of patients with primary aldosteronism: An endocrine society clinical practice guideline. J Clin Endocrinol Metab 93: 3266-3281.

4. Funder JW, Carey RM, Mantero F, Murad MH, Reincke M, et al. (2016) The management of primary aldosteronism: Case detection, diagnosis, and treatment: An endocrine society clinical practice guideline. J Clin Endocrinol Metab 101: 1889-1916.

5. Nishikawa T, Omura M, Satoh F, Shibata H, Takahashi K, et al. (2011) Guidelines for the diagnosis and treatment of primary aldosteronism. The Japan Endocrine Society 2009. Endocr J 58: 711-721.

6. Biglieri EG, Irony I, Kater CE (1989) Identification and implications of new types of mineralocorticoid hypertension. J Steroid Biochem 32: 199-204.

7. Sonoyama T, Sone M, Miyashita K, Tamura N, Yamahara K, et al. (2011) Significance of adrenocorticotropin stimulation test in the diagnosis of an aldosterone-producing adenoma. J Clin Endocrinol Metab 96: 2771-2778.

8. Young WF, Stanson AW, Thompson GB, Grant CS, Farley D, et al. (2004) Role for adrenal venous sampling in primary aldosteronism. Surgery 136 : 1227-1235.

9. Küpers EM, Amar L, Raynaud A, Plouin PF, Steichen O (2012) A clinical prediction score to diagnose unilateral primary aldosteronism. J Clin Endocrinol Metab 97: 3530-3537.

10. Artega E, Klein R, Biglieri EG (1985) Use of the saline infusion test to diagnose the cause of primary aldosteronism. Am J Med 79: 722-728.

11. Nanba K, Tsuiki M, Umakoshi H, Nanba A, Hirokawa, et al. (2015) Shortened saline infusion test for subtype prediction in primary aldosteronism. Endocrine 50: 802-806.

12. Nanba K, Tamanaha T, Nakao K, Kawashima ST, Usui T, et al. (2012) Confirmatory testing in primary aldosteronism. J Clin Endocrinol Metab 97: 1688-1694.

13. Rossi GP, Auchus RJ, Brown M, Lenders JW, Naruse M, et al. (2014) An expert consensus statement on use of adrenal vein sampling for the subtyping of primary aldosteronism. Hypertension 63: 151-160.

14. Riester A, Fischer E, Degenhart C, Reiser MF, Bidlingmaier M, et al. (2014) Age below 40 or a recently proposed clinical prediction score cannot bypass adrenal venous sampling in primary aldosteronism. J Clin Endocrinol Metab 99: E1035- E1039.

15. Jiang Y, Zhang C, Wang W, Su T, Zhou W, et al. (2015) Diagnostic value of ACTH stimulation test in determining the subtype of primary aldosteronism. J Clin Endocrinol Metab 100: 1837-1844.

16. Terui K, Nigawara T, Kageyama K, Moriyama T, Sakihara S, et al. (2016) Evaluation of the (1-24) adrenocorticotropin stimulation test for the diagnosis of primary aldosteronism. J Renin Angiotensin Aldosterone Syst 17: 1-6. 
Citation: Tsushima Y, Terui K, Makita K, Asari Y, Ishigame N, et al. (2017) Post-Saline Infusion Plasma Aldosterone Concentrations are Well Correlated with the Lateralized Ratio of Adrenal Venous Sampling in Patients of Primary Aldosteronism. J Steroids Horm Sci 8: 186. doi:10.4172/2157-7536.1000186

Page 6 of 6

17. Magill SB, Raff H, Shaker JL, Brickner RC, Knechtges TE, et al. (2001) Comparison of adrenal vein sampling and computed tomography in the differentiation of primary aldosteronism. J Clin Endocrinol Metab 86: 1066-1071.

18. Weigel M, Riester A, Hanslik G, Lang K, Willenberg HS, et al. (2015) Post-saline infusion test aldosterone levels indicate severity and outcome in primary aldosteronism. Eur J Endocrinol 172: 443-450.

19. Li Y, Liu Y, Li J, Wang X, Yu Y (2016) Sodium infusion test for diagnosis of primary aldosteronism in Chinese population. J Clin Endocrinol Metab 101: 89-95.

20. Cornu E, Steichen O, Nogueira-Silva L, Küpers E, Pagny JY, et al. (2016) Suppression of aldosterone secretion after recumbent saline infusion does not exclude lateralized primaryaldosteronism. Hypertension 68: 989-994.
21. Satoh F, Abe T, Tanemoto M, Nakamura M, Abe M, et al. (2007) Localization of aldosterone-producing adrenocortical adenomas: Significance of adrenal venous sampling. Hypertens Res 30: 1083-1095.

22. Umakoshi H, Naruse M, Wada N, Ichijo T, Kamemura K, et al. (2016) Adrenal venous sampling in patients with positive screening but negative confirmatory testing for primary aldosteronism. Hypertension 67: 1014-1019.

23. Dekkers T, Prejbisz A, Schultze Kool LJ, Groenewoud HJ, Velema M, et al. (2016) Adrenal vein sampling versus CT scan to determine treatment in primary aldosteronism: An outcome-based randomized diagnostic trial. Lancet Diabetes Endocrinol 4: 739-746. 